\title{
Original
}

\section{Análisis comparativo de la mortalidad del médico en Costa Rica, 1950-2003}

\author{
Mayra Cartín-Brenes ',Yahaira On-Cubillo ${ }^{2}$, Francini Varela-Vindas ${ }^{3}$
}

\section{Resumen}

Justificación y objetivo: En nuestro país, los médicos tienen la creencia de que, a causa del tipo de profesión que ejercen, gozan de una vida más corta y experimentan una mayor mortalidad que la población general. Existen pocas investigaciones realizadas que sugieran una sobremortalidad de los médicos y ninguna que realice un análisis comparativo con otros profesionales agremiados. Considerando que los recursos que se invierten para la formación de profesionales en el área son muy altos, la muerte prematura en este grupo implicaría un costo social muy elevado. En esta investigación se obtuvo y se analizó información de la mortalidad del médico en Costa Rica, para el período 1950 - 2003, por medio de la cual se permite conocer su comportamiento en el gremio médico nacional, en relación con el de otros profesionales y el de la población general.

Métodos: Se comparó la población de médicos con la de farmacéuticos, abogados e ingenieros y arquitectos, durante el período en estudio. Se construyeron las bases de datos. Se caracterizaron las poblaciones y se analizó el comportamiento de la mortalidad en ellas a través de diferentes métodos estadísticos

Resultados: En cuanto a los resultados más relevantes, se obtuvo que las cuatro poblaciones de profesionales son similares en cuanto a su crecimiento, medias de edad de incorporación (28 años) y edad de muerte (58 años). No obstante, difieren en su distribución por sexo; conforme ha pasado el tiempo más mujeres se han incorporado en estas carreras. El porcentaje de muertes

1. Epidemióloga y demógrafa. Universidad de Costa Rica.

2. Especialista en Medicina Familiar y Comunitaria. Caja Costarricense de Seguro Social.

3. Especialista en Medicina Familiar y Comunitaria. Caja Costarricense de Seguro Social.

Abreviaturas: REM, razón estandarizada de mortalidad; I.C, Intervalo de confianza; AVPP, años de vida potencialmente perdidos.

Correspondencia: Dra. Yahaira On Cubillo, Correo electrónico: yoc@ispp.net ó yahaira.on@gmail.com

ISSN 0001-6002/2006/48/3/123-128 Acta Médica Costarricense, $@ 2006$ Colegio de Médicos y Cirujanos ocurridas antes de alcanzar la esperanza de vida respectiva fue similar en todos los grupos ( $90 \%)$. La razón estandarizada de mortalidad (REM), es similar en los cuatro grupos y menor que la de la población general. La mortalidad ha disminuido a través del tiempo y el riesgo de morir en la actualidad es menor que en generaciones previas. La supervivencia entre los incorporados de 20 a 29 años, es mayor en las médicas y en los médicos especialistas, con respecto a quienes ejercen la medicina general. Es importante señalar que no se encontró diferencia en la supervivencia por especialidad ni por profesión. Las enfermedades cardiovasculares y el cáncer en los cuatro grupos estudiados se determinan como las principales causas de muerte.

Conclusión: La mortalidad de los profesionales es menor que la de la población general, lo cual concuerda con otras investigaciones según las cuales la mortalidad es menor en los grupos de mayor educación. En los grupos de profesionales analizados, la mortalidad es similar y ha disminuido a través del tiempo, siendo que las causas de muerte en los profesionales son las mismas que en la población en general.

Descriptores: médico, mortalidad, cohorte, supervivencia, causas de muerte.

Key words: physicians, mortality survival, cohorte, causes of death.

Recibido: 21 de octubre de 2006 Aceptado: 28 de marzo de 2006 
El estudio tiene como propósito describir el comportamiento de la mortalidad del médico en Costa Rica, incorporado desde 1950 hasta el año 2003, y realizar un análisis comparativo de esta en relación con la de otros profesionales.

La comunidad médica costarricense ha mantenido la creencia, desde hace mucho tiempo, de que por sus largas jornadas de trabajo en condiciones de estrés, sus miembros tienen una vida más corta que la de la población en general. Esto probablemente se deba a la confusión existente entre la edad a la que mueren hoy los médicos y la esperanza de vida que presenta Costa Rica en la actualidad. Si un galeno muere a los de 65 años, da la impresión de que, al no llegar a los 78 años de esperanza de vida que ostenta Costa Rica, se está frente a una muerte prematura. Esta concepción es errada. Si una persona muere de 65 años en 2005, a ese individuo se le debe comparar con la esperanza de vida al nacer que existía cuando nació; por ejemplo, una persona de 65 años en 2005, nació en 1940, cuando la esperanza de vida era de 46,7 años, por lo que si muere de 65 años, esa persona está sobre el promedio de edad que vivieron los nacidos en 1940 (Cuadro 1).

Es importante recordar el concepto de esperanza de vida, como la cantidad de años en promedio que se espera que una persona viva a partir de su nacimiento, si las condiciones de mortalidad se mantuviesen.

En otros países, al igual que en Costa Rica, en algún momento se ha tenido la misma inquietud ${ }^{1}$, sin embargo, estudios realizados arrojan resultados contradictorios. Mientras que unos evidencian una muerte prematura del médico y una mayor tasa de mortalidad ${ }^{2-5}$, otros concluyen que el médico disfruta de una mayor longevidad en comparación con otros grupos poblacionales ${ }^{6-10}$.

Las investigaciones de mortalidad efectuadas en el país sugieren una sobremortalidad de los médicos, no obstante, esto no se ha logrado comprobar, porque los datos existentes no han sido suficientemente analizados ${ }^{11-13} \mathrm{y}$, además, no se cuenta con estudios que comparen su mortalidad con la de otros profesionales. Es por ello, que en nuestro análisis, el primer paso consistió en determinar si el médico en Costa Rica realmente tiene una vida más corta, para luego establecer si el comportamiento difiere del de otros grupos profesionales.

Los datos analizados en el estudio son: sexo, edad, profesión, fecha de incorporación al colegio profesional, fecha de defunción y causa de muerte.

\section{Materiales y Métodos}

Inicialmente se identificó y seleccionó el grupo de comparación (farmacéuticos, abogados e ingenieros y arquitectos) para construir las bases de datos utilizadas.

El proceso de selección requirió analizar previamente la información disponible en los colegios profesionales.

Se realizó una caracterización de las poblaciones en cuanto a su crecimiento, distribución por sexo y edad de incorporación al colegio profesional respectivo, según los siguientes criterios de presentación:

- Medidas de tendencia central, posición y dispersión elementales: promedio, mediana, moda e intervalo intercuartílico de la edad de incorporación

- Crecimiento de las poblaciones en estudio a partir de 1950, por quinquenios de incorporación.

- Distribución por sexo y análisis de la razón de masculinidad de cada población a inicio, mitad y fin del período en estudio (1950-1974; 1975-1999 y 20002003).

Se analizó la mortalidad de acuerdo con varios métodos estadísticos $^{14-17}$ (en el Cuadro 2 se explica cada uno de los análisis):

- Uso de medidas de tendencia central, posición y dispersión, para caracterizar la mortalidad por edad de defunción y años laborados por los diferentes profesionales

- Cálculo de los años de vida potencialmente perdidos de los profesionales fallecidos

\begin{tabular}{|cccccc|}
\hline \multicolumn{6}{|c|}{ Cuadro 1. Esperanza de vida al nacer, según década de nacimiento de los profesionales en estudio } \\
\hline $\begin{array}{c}\text { Década de } \\
\text { nacimiento }\end{array}$ & $\begin{array}{c}\text { Esperanza de vida } \\
\text { al nacer }\end{array}$ & $\begin{array}{c}\text { Edad promedio } \\
\text { de incorporación }\end{array}$ & $\begin{array}{c}\text { Año promedio } \\
\text { de incorporación }\end{array}$ & $\begin{array}{c}\text { Edad promedio } \\
\text { al morir }\end{array}$ & $\begin{array}{c}\text { Cantidad de } \\
\text { defunciones }\end{array}$ \\
\hline 1920 & 34,15 & 39 & 1959 & 72 & 3 \\
1930 & 42 & 36 & 1966 & 68 & 9 \\
1940 & 46,7 & 29 & 1969 & 53 & 5 \\
1950 & 55,6 & 27 & 1977 & 44 & 2 \\
1960 & 62,6 & 28 & 1988 & 41 & 2 \\
1970 & 65,7 & 26 & 1996 & 29 & 2 \\
\hline
\end{tabular}

Fuente: Revisión bibliográfica y bases de datos construidas, 2004. 


\begin{tabular}{|c|c|}
\hline \multicolumn{2}{|r|}{ Cuadro 2. Métodos estadísticos utilizados } \\
\hline Método estadístico & Definición \\
\hline $\begin{array}{l}\text { Años de vida } \\
\text { potencialmente } \\
\text { perdidos }\end{array}$ & $\begin{array}{l}\text { Sirve para medir el impacto de la muerte prematura en los diferentes grupos. Este indi- } \\
\text { cador se calcula a través de la diferencia entre la esperanza de vida (tomada de las tablas } \\
\text { de vida del CCP) y la edad de muerte de cada profesional fallecido. } \\
\qquad \text { AVPP = Esperanza de vida - Edad de muerte }\end{array}$ \\
\hline $\begin{array}{l}\text { Razón estandarizada } \\
\text { de mortalidad }\end{array}$ & $\begin{array}{l}\text { Método indirecto de ajuste de tasas, que permite estimar el número de defunciones (la } \\
\text { cantidad de defunciones que se espera) que hubieran ocurrido en esos grupos si los habi- } \\
\text { tantes que los componen se murieran de acuerdo con la misma tasa de mortalidad de la } \\
\text { población estándar. La suma de las defunciones esperadas produce un total de defun- } \\
\text { ciones esperadas en cada población comparables con las defunciones observadas por } \\
\text { medio de una razón: } \\
\qquad \text { REM }=\frac{\text { Muertes observadas }}{\text { Muertes esperadas }} 100\end{array}$ \\
\hline $\begin{array}{l}\text { Mortalidad acumulada } \\
\quad \text { por cohortes }\end{array}$ & $\begin{array}{l}\text { Una cohorte es una población expuesta a un determinado evento que se observa a través } \\
\text { del tiempo, para investigar su aparición. Cabe recalcar que este método no considera la } \\
\text { estructura por edad de las poblaciones. Los sujetos ingresan al estudio con base en el } \\
\text { año de incorporación y se siguen por períodos quinquenales hasta el evento final (la } \\
\text { muerte). }\end{array}$ \\
\hline $\begin{array}{l}\text { Cohortes de } \\
\text { nacimiento }\end{array}$ & $\begin{array}{l}\text { Agrupa la población en función de la generación a la que pertenecen. Muestra la tenden- } \\
\text { cia en mortalidad conforme envejecen las mismas personas. }\end{array}$ \\
\hline $\begin{array}{l}\text { Análisis de } \\
\text { supervivencia }\end{array}$ & $\begin{array}{l}\text { El método de Kaplan-Meier es el de elcción para este estudio, ya que se trabaja con un } \\
\text { número pequeño de sujetosen los que se conocen los tiempos exactos de las muertes, } \\
\text { los casos no entran al estudio en un mismo tiempo y se analizan los datos antes de que } \\
\text { todos hayan presentado el evento, es decir la muerte. Además permite estimar inmedi- } \\
\text { atamente después de cada muerte la probabilidad de supervivencia. }\end{array}$ \\
\hline
\end{tabular}

- Determinación de la razón estandarizada de mortalidad de las diferentes poblaciones

- Análisis de la mortalidad de los médicos por cohorte de nacimiento

- Análisis de la supervivencia de los médicos según sexo, tipo de ejercicio profesional y especialidad

- Identificación y distribución porcentual de las principales causas de muerte de las diferentes poblaciones en estudio

El análisis estadístico se realizó con hojas de cálculo Excel y Epi-Info 2002.

\section{Resultados}

El crecimiento de las poblaciones de profesionales ha sido similar durante el período en estudio. Al principio, en las profesiones analizadas predominaba el sexo masculino, sin embargo, conforme ha pasado el tiempo se observa como la mujer ha aumentado su representación en estos gremios, llegando incluso a predominar en el grupo de los farmacéuticos y de los abogados.

La media de la edad de incorporación al colegio profesional es similar en las diferentes profesiones (27 a 31 años).

Un dato importante es que el promedio de la edad de muerte es prácticamente el mismo en todos los profesionales de los cuatro grupos de interés (58 años, figura 1).

Según los años laborados, se obtiene que el valor central oscila entre los 21 y los 26 años en los cuatro grupos, aunque el comportamiento difiere entre las distintas profesiones.

En cuanto al cálculo de los años de vida potencialmente perdidos, se encuentra que el porcentaje de muertes ocurridas antes de alcanzar la esperanza de vida respectiva fue similar en todos los grupos ( 90\%), dato que es de esperar por tratarse de poblaciones jóvenes. 
Los cálculos de la razón estandarizada de mortalidad (REM) con su respectivo intervalo de confianza al 95\%, de los cuatro grupos de profesionales estudiados, se presentan en el Cuadro 3 y Figura 2, donde se puede observar que el valor de la REM + I.C. es inferior a 100 y similar entre los cuatro grupos, lo cual se interpreta como que la mortalidad en los profesionales es menor que en la población general, sin que haya diferencia estadísticamente significativa entre los distintos tipos de profesión analizados.

En el análisis de la mortalidad por cohorte de nacimiento de los médicos, se concluye que esta ha disminuido a través del tiempo y que en la actualidad el riesgo de morir a temprana edad es menor que en generaciones previas (Cuadro 4, Figura 3).

El análisis de supervivencia en los médicos incorporados entre los 20 y los 29 años muestra que existe una mayor supervivencia en las mujeres $(\mathrm{p}=0,01)$, así como en los médicos especialistas con respecto a los generales $(\mathrm{p}=0,00)$. Sin embargo, no se encontró diferencia en las curvas de supervivencia por especialidad $(0,97)$, ni al comparar los médicos con los otros profesionales $(\mathrm{p}=0,10)$ Figura 4.

En los cuatro grupos profesionales, las principales causas de muerte fueron las enfermedades cardiovasculares y el cáncer.

\section{Discusión}

El comportamiento de la mortalidad de las cuatro poblaciones estudiadas durante los últimos 53 años es similar. La cuarta parte de las muertes ocurrió en menores de 50 años, de hecho, la edad promedio de muerte es de 58 años, la cual es una edad temprana. Sin embargo, al comparar los promedios de edad de muerte con la esperanza de vida correspondiente al año de nacimiento, se observa que es a partir de los nacidos en 1950, que las muertes suceden antes de que se logre alcanzar la esperanza de vida respectiva. Este fenómeno es de esperar, debido a que se estudió un período de 53 años, durante el cual se han incorporado personas que en este momento son jóvenes y que en su mayoría están aún vivas. Ello indica que, al estar analizando poblaciones jóvenes, en las cuales a pesar de que el riesgo de morir es menor, las muertes que se suscitan se presentan a temprana edad, sin que esto implique que su mortalidad sea precoz.

Con base en las consideraciones anteriores, para lograr eliminar el sesgo que se produce en la estructura por la edad de las poblaciones, se utilizó la razón estandarizada de mortalidad, la cual estima la cantidad de defunciones que se hubiesen producido en los grupos de profesionales, si ellos tuvieran la misma probabilidad de morir que la población costarricense. Si la razón estandarizada de mortalidad es

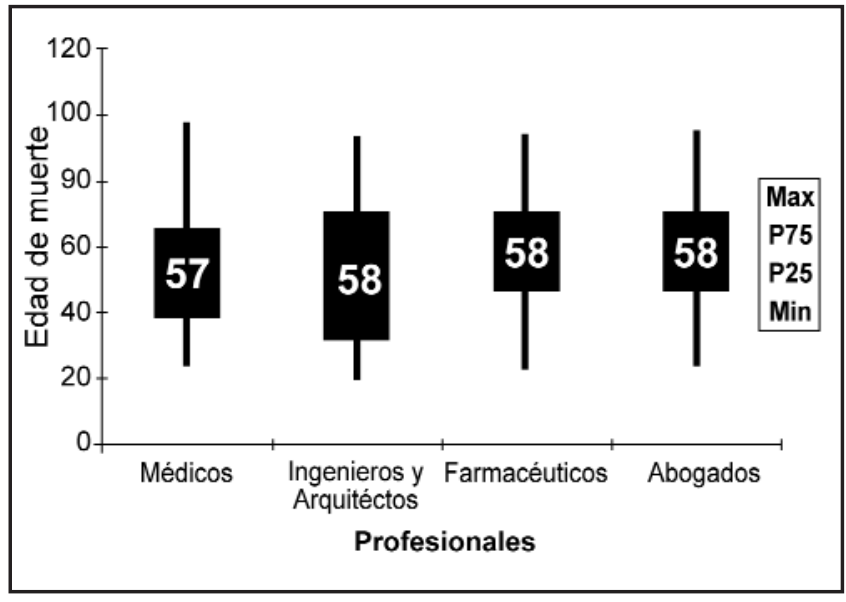

Figura 1. Medidas de tendencia central, posición y dispersión de la edad de muerte de los profesionales. Costa Rica, 1950-2003.

mayor que 100, significa que la cantidad de defunciones en ese grupo profesional fue mayor que la esperada. Este es un indicador más estable y permite realizar comparaciones entre poblaciones pequeñas y la población general.

Se calcularon las muertes esperadas a partir de las tasas de mortalidad del país, por grupo de edad durante el período de estudio (1976-2003); se eligió a partir del año 1976, en que se inician las defunciones observadas en los grupos de profesionales. El efecto mostró que la REM fue menor a 100 para todos los grupos profesionales.

Al seguir a través del tiempo la cohorte de nacimiento de los médicos (1946-1954) que tenía 20-24 años en 19701974, y compararla con generaciones previas, se observa como la mortalidad es similar hasta los 45 años de edad, en cuyo caso la mortalidad de la cohorte en análisis es menor que la de generaciones anteriores, lo cual indica que actualmente el riesgo de morir es menor que en las generaciones previas.

El análisis de supervivencia revela que el comportamiento de la mortalidad en las cuatro poblaciones es similar. Los hallazgos significativos se obtuvieron en el grupo de incorporados entre los 20-29 años, lo cual es relevante si se considera que el promedio de edad de incorporación al colegio profesional es de 28 años. Específicamente en el gremio médico, las mujeres sobreviven más que los hombres y en estos últimos los especialistas sobreviven más que los generalistas. Sin embargo, al analizar la supervivencia por especialidad, no hay diferencias. Cabe mencionar que el $68 \%$ de los hombres se incorporan a la profesión de medicina en dicho rango de edad (28 años) y que el promedio de años laborados de los fallecidos de este grupo es de 22,96 para los especialistas y 17,04 para los generalistas. Esto demuestra que tales hallazgos merecen ser estudiados, para lo cual se deberían considerar las condiciones de vida que podrían estar influyendo. 


\begin{tabular}{|lcccc|}
\hline \multicolumn{3}{|c|}{$\begin{array}{c}\text { Cuadro 3. Razón estandarizada de mortalidad } \\
\text { de los profesionales, Costa Rica, }\end{array}$} \\
\hline $\begin{array}{l}\text { Profesionales } \\
\text { esperadas }\end{array}$ & $\begin{array}{c}\text { Muertes } \\
\text { observadas }\end{array}$ & $\begin{array}{c}\text { Muertes } \\
\text { REM }\end{array}$ & $\begin{array}{c}\text { Intervalos } \\
\text { de confianza }\end{array}$ \\
\hline $\begin{array}{l}\text { Médicos } \\
\text { Ingenieros }\end{array}$ & 325 & 233 & 72 & $62,48-80,9$ \\
y arquitectos & 425 & 288 & 68 & $60,08-75,76$ \\
Farmacéuticos & 159 & 92 & 58 & $46,04-69,68$ \\
Abogados & 560 & 351 & 63 & $56,09-69,2$ \\
\hline Fuente: CCP y bases de datos construidas, 2004 & & \\
\hline
\end{tabular}

\section{Agradecimientos y colaboradores}

A la Doctora Nuria Báez Barahona, Coordinadora del Posgrado Medicina Familiar y Comunitaria, por impulsarnos y apoyarnos para realizar esta investigación.

\section{Abstract}

Justification and Objective: In our country, physicians tend to share the belief that, due to the type of profession they exert, they live shorter life spans and experience a higher mortality rate than the general population. There are few studies made in our country that suggest and none which features a comparative analysis with other professions. Considering the cost invested in the formation of professionals in this area, premature deaths in this group may imply a very high cost for the society as a whole.

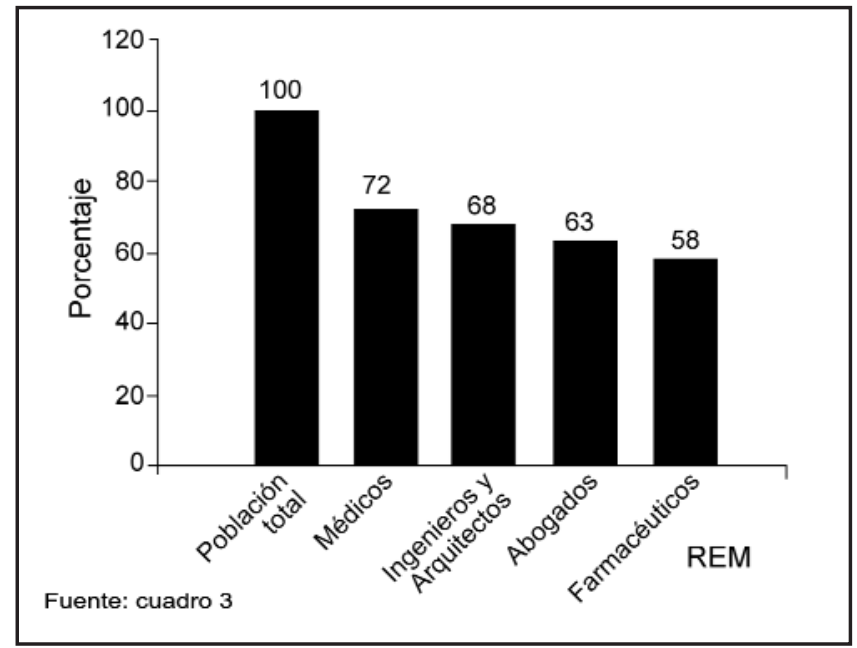

Figura 2. Razón estandarizada de mortalidad para los diferentes grupos de profesionales.

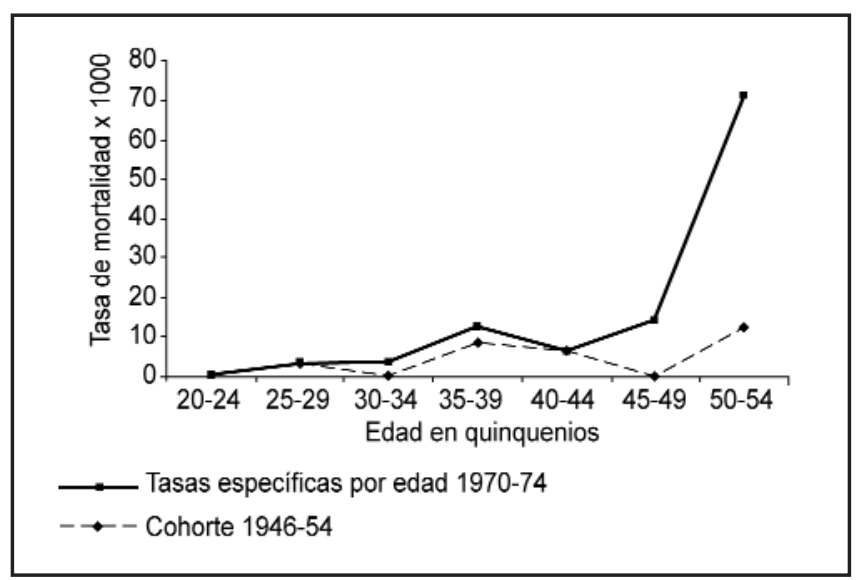

Figura 3. Tasas de mortalidad específicas por edad de los médicos, 1970-1974, y cohorte de médicos en 1946-1954. Costa Rica.

\begin{tabular}{|c|cccccccc|}
\hline \multicolumn{8}{|c|}{ Cuadro 4. Tasas de mortalidad específicas por edad de los médicos. Costa Rica, 1970-2003 } \\
\hline $\begin{array}{c}\text { Edad en } \\
\text { quinquenios }\end{array}$ & $1970-74$ & $1975-79$ & $1980-84$ & $\begin{array}{c}\text { Año } \\
1985-89\end{array}$ & $1990-94$ & $1995-99$ & $2000-03$ \\
\hline \multirow{2}{*}{$20-24$} & 0,00 & 0,00 & 0,00 & 0,00 & 0,00 & 0,00 & 0,00 \\
$25-29$ & 0,00 & 3,13 & 1,46 & 1,82 & 2,29 & 1,30 & 0,84 \\
$30-34$ & 3,17 & 0,00 & 0,00 & 1,18 & 6,10 & 3,63 & 4,36 \\
$35-39$ & 4,12 & 2,80 & 0,00 & 8,37 & 7,91 & 4,32 & 6,86 \\
$40-44$ & 0,00 & 18,94 & 0,00 & 9,12 & 6,16 & 4,44 & 4,29 \\
$45-49$ & 14,49 & 6,21 & 11,19 & 8,38 & 15,15 & 0,00 & 8,88 \\
\hline $50-54$ & 58,82 & 14,29 & 6,29 & 29,85 & 14,20 & 15,24 & 12,32 \\
\hline
\end{tabular}




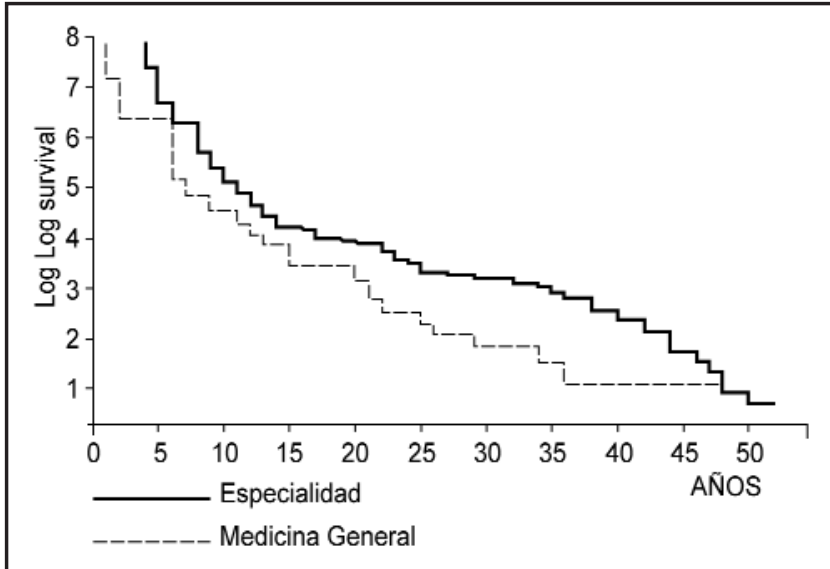

Figura 4. Análisis de supervivencia de los médicos de sexo masculino, incorporados entre los 20-29 años, según tipo de ejercicio profesional..Costa Rica, 1950-2003.

From this study we now know mortality behavior within the medical profession and its comparison with other professions and the general population.

Methods: The population of physicians was compared to that of pharmacists, lawyers, engineers and architects during the time of the study. Data bases were created. Mortality behaviour was analysed using different statistical methods.

Results: The 4 populations under study were similar in their growth patterns, average age of incorporation to their professional associations (28 years) and age of death (58 years). However their distribution according to gender has varied, since in recent years more women have become professionals in these careers.

The percentage of deaths that occurred before reaching their respective life expectancy was similar for all groups ( $90 \%$ ). The standardized mortality ratio (SMR) was similar for all groups and lower than that for the general population. Mortality rates have decreased throughout time and the risk of death today is lower than that of previous generations. Survival rates in those who recently joined their professional associations (age 20 to 29) were higher in female physicians and in medical specialists than in general practitioners. There was no difference in survival rates by specialty or by profession. Cardiovascular disease and cancer were the leading causes of death for all groups.

Conclusions: Mortality rates among professionals are lower than that of the general population, this coincides with findings of previous investigations that have shown that mortality rates are lower in more educated populations. Within the professional groups analyzed, mortality rates are similar and have decreased throughout time, being the leading causes of death in these groups the same as for the general population.

\section{Referencias}

Williams SU, Munford RS, Colton T, Murphy DA, Poskanser DC. Mortality among physicians: a cohort study. J Chronic Dis, 1971; 24: 391-401.

2. Copplestone JF, Rose RJ. Occupational mortality among male population, other than Maori, 20-64yr of age based on deaths, 1959-1963 and population census, 1961. Special Report N28, New Zealand National Health Statistics Center, 1967.

3. King H. Health in the medical and other learned professions. J Chronic Dis, 1970; 23: 257-281.

4. Lindhardt, $\mathrm{M}$ et al. Causes of death among the medical profesión. Dan Med Bull, 1963; 10:59-64.

5. Ogle W. Statistics of mortality in the medical profession. Med Chir Trans 1886; 69: 217-237.

6. Dean G. The causes of death of South African doctors and dentists. SAF Med J, 1969; 43: 495-500.

7. Frank E, Biola H, Burnett CA. Mortality rates and causes among U.S. physicians. Am J Prev Med, 2000; 19: 155-159.

8. Goodman J. Longevity and mortality of American physicians, 196973, MMFQ Summer, 1975; 353-75.

9. Guralnick L. Mortality by occupation and industry among men 20-64 yr of age, United States, 1950. Vital Statistics-Special Reports. Washington National Vital Statistics Division, Public Health Service, US. Department of Health, Education, and Welfare, 1963; 53.

10. Registral general for england and wales: mortality of men in certain occupations in the three years 1910, 1911 and 1912. Supplement to the 75th Annual Report of the Registrar-General for England and Wales. Part IV. London, HMSO, n.d.

11. Castro J. Un análisis de mortalidad médica por edades en Costa Rica. Opinión Médica 1992; 10: 13-14.

12. Valverde, C. Edad de muerte de los médicos en Costa Rica. Opinión Médica 1992; 10: 20.

13. Jiménez M. La mortalidad en el cuerpo médico nacional. Acta Méd Costarric 1997; 39: 38-43.

14. Dawson B, Trapo R. Bioestadística médica. (2ed) México: Manual Moderno, 1997: 223-248.

15. Lilienfeld A, Lilienfeld D. Fundamentos de Epidemiología. Editorial Adison Weslwy Iberoamericana S.A. 1983; 57-112.

16. Centro para el Control y Prevención de Enfermedades. Principios de epidemiología: Una introducción a la epidemiología y bioestadística aplicadas. Georgia, 1992.

17. Dos Santos Isabel. Epidemiología del cáncer: principios y métodos. Introducción al análisis de la supervivencia. Agencia internacional de investigaciones sobre el Cáncer, OMS. Francia, 1999. 